\title{
Adaptive 3D ray tracing approach for indoor radio signal prediction at $3.5 \mathrm{GHz}$
}

\author{
Mohd Nazeri Kamaruddin ${ }^{1}$, Tan Kim Geok ${ }^{1}$, Omar Abdul Aziz ${ }^{2}$, Tharek Abd Rahman², \\ Ferdous Hossain ${ }^{1}$, Azlan Abdul Aziz ${ }^{1}$ \\ ${ }^{1}$ Faculty of Engineering and Technology, Multimedia University, Melaka, Malaysia \\ ${ }^{2}$ Faculty of Electrical Engineering, Universiti Teknologi Malaysia, Johor Bahru, Malaysia
}

\begin{tabular}{l} 
Article Info \\
\hline Article history: \\
Received Mar 26, 2021 \\
Revised Jul 19, 2021 \\
Accepted Aug 14, 2021 \\
\hline Keywords: \\
Measurement campaign \\
Path loss \\
Radio signal prediction \\
Ray tracing \\
Simulation
\end{tabular}

Corresponding Author:

Mohd Nazeri Kamaruddin

Faculty of Engineering and Technology, Multimedia University

Ayer Keroh Lama Street, 75450 Melaka, Malaysia

Email: nazeri.kamaruddin@mmu.edu.my

\begin{abstract}
This paper explained an adaptive ray tracing technique in modelling indoor radio wave propagation. As compared with conventional ray tracing approach, the presented ray tracing approach offers an optimized method to trace the travelling radio signal by introducing flexibility and adaptive features in ray launching algorithm in modelling the radio wave for indoor scenarios. The simulation result was compared with measurements data for verification. By analyzing the results, the proposed adaptive technique showed a better improvement in simulation time, power level and coverage in modelling the radio wave propagation for indoor scenario and may benefit in the development of signal propagation simulators for future technologies.
\end{abstract}

This is an open access article under the CC BY-SA license.

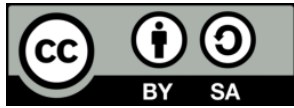

\section{INTRODUCTION}

The fast progression of wireless communications network systems within the last few years has become a growing need to advance and efficient computerized tools for radio wave propagation prediction in various environments [1]. The massive usage of smart gadgets such as laptops, tablets, smart phones, and smart watches, has continuously contributed to the advancement of wireless communication technologies to accommodate the demand in the market. Therefore, a smart radio wave propagation prediction model plays an important role in the analysis of wireless communication systems especially for the emerging smart city design, upgrading the 4G mobile networks, internet of things (IoT), and the evaluation of 5G network system [2]. Radio propagation is a foundation to analyze and predict the signal details [3]. Direct measurement is one of the methods to evaluate the onsite performance, but it needs a considerable amount of time and very costly. On the other way, the analysis can be performed by a computerized simulation tool that offers more dynamic, lowcost, and more efficient [4].

In recent decades, radio frequency $(\mathrm{RF})$ propagation models have been one of the major interesting research subjects due to the rapid development of new wireless communication system. Some propagation models have been proposed to predict the propagation paths of the radio signals and determining the optimal locations of the base stations [5], [6]. Every approach is determined by different parameters such as coverage area, bandwidth, and types of environments. For bandwidth, the models can be classified as either wideband or narrowband. The wideband models can be further divided into arrival times of rays, power delay profile or tapped delay line approaches [7]. At the same time, narrowband models can be grouped into different types 
based on path loss and large-scale or small-scale fading. In term of surrounding conditions, there are two major classifications which is either outdoor or indoor propagation. For indoor environment, the propagation models are grouped into stochastic, empirical or site specific models. Empirical models are derived from certain propagation parameters such as the operating frequency, the distance between the transmitter (Tx) and receiver $(\mathrm{Rx})$, and the height of the antenna [8]. These variables are assumed to follow the regular experimental data from measurement campaigns. They usually represent a set of equations derived from extensive field measurements, simple and efficient to use and suitable for environments with the same characteristics. The implementation of this model is easier as the major parameters are only the Tx-Rx distance and the operational frequency that contribute to a lower computational load. The main shortcoming of this model is lower accuracy as the propagation environment has been generalized and not well defined. For example, the empirical model for macrocells cannot be used for indoor picocells because of its range specific and not site specific.

In the stochastic approach, radio signal is modeled as random variables [9]. Similar to the empirical model, the precision is also lower for signal prediction as the details of the propagation environment are not considered. On the other hand, for site-specific models, Maxwell's equations are used in the radio wave propagation prediction. In these models, the precision of the simulation result can be improved. The simulation environment is well defined, especially in launching and tracing the ray, which makes the radio wave propagation to be more accurate and practical [10]. However, due to the increase in the detail of the environment features and ray propagation to be calculated, most of the site-specific models require higher computational load and simulation time, which contributes to the main shortcoming of this approach. In order to perform radio propagation analysis, the ray tracing (RT) which belongs to site-specific models is the best option to generate the practical result [11]. For indoor environment, ray tracing technique is the most capable technology to increase the accuracy of the simulation results [12].

The efficiency of ray tracing method depends on its capability to simulate the real surrounding of the tested scenario and trace the path traveled by each ray from the transmitting base station. However, the complexity of the simulation will become higher as more detailed items in the environment are to be considered to increase the accuracy. The computational load to trace the path and other propagation mechanisms will be increased significantly [13]. There are a lot of methods studied by many radio propagation researchers, but only a few concentrates on the analysis of the adaptive step size for ray launching that may contribute to the optimization in modelling the indoor radio wave propagation. In this study, our proposed adaptive threedimensional (3D) ray tracing was analyzed together with renowned 3D ray tracing using the shooting and bouncing (SB) approach. All the modelings and simulations are applied using an in-house developed simulator. The actual measurement layouts and experimental parameters were used in simulations for result verification and validation. The operating frequency of $3.5 \mathrm{GHz}$ was chosen for this study, which belongs to the sub- $6 \mathrm{GHz}$ band for $5 \mathrm{G}$ technologies [14]. For comparison, the 3D shooting and bouncing RT was selected which is the most used technique in radio propagation modeling [15], [16]. This technique has been further expanded and used extensively for modeling radio propagation in multipath channels [17], [18]. Based on our analysis of the simulation results, the adaptive $3 \mathrm{D}$ ray tracing methods produced an accurate propagation prediction that may benefit the design of the simulator to analyze radio frequency signal propagation.

\section{RAY CONCEPT}

In the ray concept, every ray follows the nature of sunlight that is assumed to propagate from a source point and extends infinitely along a straight line. The simple way to obtain the power level of radio signal propagation in free space is by using Friis's formula as shown in (1).

$$
\frac{P_{r}}{P_{t}}=G_{t} G_{r}\left(\frac{\lambda}{4 \pi r}\right)^{2}
$$

In (1), $P_{t}, P_{r}, G_{t}, G_{r}, \lambda$, and $r$ represent the transmitted power, power level, transmitter antenna gain, receiver antenna gain, wavelength, and separation distance between the transmitter and the receiver, respectively. However, in ray theory, Maxwell's equations are applied extensively in radio propagation modelling. The electric field is represented by (2) [19].

$$
\bar{E}(\bar{r})=\bar{e}(\bar{r}) e^{-j \beta_{0} S(\bar{r})}
$$

The symbol $\bar{e}(\bar{r})$ denotes the magnitude vector, $\bar{S}(\bar{r})$ refers to propagation path, and the wavenumber, $\beta_{0} \rightarrow \infty$ at high frequency. The tracing of ray for radio propagation simulation can be implemented using certain principles. Most rays are assumed to travel in a straight path within the same medium before intercepting an object or obstacle [20]. The main obstacles to be considered for indoor conditions must be electrically larger 
than one wavelength. The reflection coefficient for radio propagation is calculated by Fresnel equations using the wave impedance as (3), (4):

$$
\begin{aligned}
& \Gamma_{S}=\left|\frac{Z_{2} \cos \theta_{i}-Z_{1} \cos \theta_{t}}{Z_{2} \cos \theta_{i}+Z_{1} \cos \theta_{t}}\right|^{2} \\
& \Gamma_{p}=\left|\frac{Z_{2} \cos \theta_{t}-Z_{1} \cos \theta_{i}}{Z_{2} \cos \theta_{t}+Z_{1} \cos \theta_{i}}\right|^{2}
\end{aligned}
$$

From (3) and (4), both vertical and horizontal polarizations reflection coefficients are represented by $\Gamma_{S}$ and $\Gamma_{p}$, respectively. The reflected and incident angles are denoted by $\theta_{t}$ and $\theta_{i}$. The impedances of the first and second mediums are denoted by $\mathrm{Z}_{1}$ and $\mathrm{Z}_{2}$ respectively. The impedance value is related to the relative permittivity of the object, $\varepsilon_{\mathrm{r}}$ and the permeability $\mu_{\mathrm{r}}$ for non-magnetic material of the building structures.

A ray is modeled as a line-of-sight $(\mathrm{LoS})$ path if it propagates directly from a source to a receiver. On the other hand, non-line-of-sight (NLoS) path is considered if a ray is hitting any object and reflected once or multiple times when propagating to the receiver. Equation (5) is used to compute the path loss (PL) for LoS and NLoS radio propagation [19].

$$
P L(f, d)[d B]=F S P L(f, 1 m)+10 n \log _{10} \frac{d}{1[m]}+X_{\sigma}
$$

By referring to (5), $n$ denotes the path loss exponent and $X_{\sigma}$ refers to a zero mean Gaussian variable pertaining to the standard deviation $\sigma$. The free space path loss (FSPL) represents the path loss for a 1-m distance between Tx and Rx, and can be calculated by (6).

$$
\operatorname{FSPL}(f, 1 \mathrm{~m})[\mathrm{dB}]=20 \mathrm{n} \log _{10} \frac{4 \pi f}{c}
$$

In this (6), $f$ denotes operating frequency while $c$ refers to the speed of light. If a ray is hitting a sharp edge, a cone of rays is generated due to the diffraction mechanism. The analysis of the diffraction mechanism is more complex. Here in this study, the diffracted rays were calculated based on the knife-edge approach in the ray tracing simulation. The power level of the diffracted ray is very low as compared to direct rays and normally considered only for the shadowed area in the simulation. By using the geometrical theory of diffraction (GTD), the Luebbers model can be used to implement diffraction in RT [21]. The diffracted power level $E_{G T D}$ is calculated using (7).

$$
E_{G T D}=E_{0} \frac{e^{-j k p \prime}}{p^{\prime}} D_{\|}^{\perp} \sqrt{\frac{p^{\prime}}{p\left(p^{\prime}+p\right)}} e^{-j k p}
$$

The symbols $E_{0}, D_{\|}^{\perp}, p^{\prime}, p, \mathrm{k}$, denotes the source point amplitude, diffraction coefficient, the distance between the diffraction point to $\mathrm{Rx}$, the Tx-Rx separation distance, and the wave number respectively. For non-conducting objects, (8) is used to obtain $D_{\|}^{\perp}$ as below. The symbols $\Gamma_{n \|}^{\perp}$ and $\Gamma_{0 \|}^{\perp}$ are the vertical and horizontal polarization reflection coefficients. The symbol $\phi$ represents forward directions, while $n \pi-$ $\phi$ denotes backward directions and $\phi^{\prime}$ refers to reverse phase. Other symbols used in (8) refer to specific equations as follows: $\left.\beta=\phi \pm \phi^{\prime}, a \pm(\beta)=2 \cos 2[(2 n \pi N \pm-\beta)] / 2\right], L=\left(p p^{\prime}\left(p+p^{\prime}\right)\right)$,

$$
\begin{aligned}
& D_{\|}^{\perp}=\frac{-e^{-j\left(k\left(p+p^{\prime}\right)+\frac{\pi}{4}\right)}}{2 n \sqrt{2 \pi k} \sin \beta}\left\{\frac{F\left(k L a^{+}\left(\phi-\phi^{\prime}\right)\right)}{\tan \left[\frac{\pi+\phi-\phi^{\prime}}{2 n}\right]}+\frac{F\left(k L a^{-}\left(\phi-\phi^{\prime}\right)\right)}{\tan \left[\frac{\pi+\phi-\phi^{\prime}}{2 n}\right]}+\right. \\
& \left.\Gamma_{0 \|}^{\perp} \frac{F\left(k L a^{-}\left(\phi+\phi^{\prime}\right)\right)}{\tan \left[\frac{\pi+\phi-\phi^{\prime}}{2 n}\right]}+\Gamma_{n \|}^{\perp} \frac{F\left(k L a^{+}\left(\phi+\phi^{\prime}\right)\right)}{\tan \left[\frac{\pi+\phi+\phi^{\prime}}{2 n}\right]}\right\}
\end{aligned}
$$

\section{PROPOSED ADAPTIVE 3D RT METHOD}

Adaptive $3 \mathrm{D}$ ray tracing is a method to simulate the propagation route of each ray using mathematical and propagation mechanisms and generates the valid paths from the transmitter to the receiver. Each valid path 
comprises of some straight lines connected based on propagation mechanism such as direct path, reflection, and diffraction. The trajectory direction to launch a ray is given by the angle, measured between the horizontal plane and a reference point, and the sub-angle, which is the azimuthal angle on a vertical plane, measured from the same reference point. The objective of this adaptive RT is to identify rays from the transmitter to the receiver in a flexible manner but efficient. It consists of four main steps: pre-ray launch, adaptive step-size calculation, updated ray launching and determination of ray reception.

- At first, the ray is emitted at a horizontal angle based on the initial step size configured initially in the simulator. When the first ray is launched from the Tx, the signal power is stored as reference power. The second ray is then launched at a vertical angle with the initial step size, which is also set in the initial configuration.

- Secondly, a new step size is calculated based on a comparison between the power level of the current launched ray and the power level of the previous ray. The increment or decrement of the new step size is determined by the adaptive step size parameter which is set on the configuration page before the simulation. The calculation of the next step size is based on these three conditions; if the power level of the current signal is less than the previous signal, the next step size is increased by one adaptive step size. If the power level of the current signal is higher than the previous signal, the next step size is decreased by one adaptive step size. If the power level of the current signal is the same as the previous signal, the next step size remains the same. The increment of the step size is limited to the maximum allowable step size set in the configuration page. On the other hand, the decrement of the step size is also limited to the predefined minimum allowable step size. These two parameters are required to prevent the step size to become zero or a very big value. The same calculation will be applied for both horizontal and vertical angles during the launching of rays from the base station.

- Thirdly, the next ray launching at updated angles is conducted after the increment by the updated step size.

- Lastly, the rays are traced throughout the simulation using the propagation principles including diffraction, reflection, and transmission to identify the valid rays that are received by the Rx.

The significant improvement of the proposed adaptive 3D RT technique is the introduction of adaptive step size which contributes to the flexibility feature on the ray launching angle. For the region with a high or significant power level, the resolution of the ray launching is higher by having a smaller step size. On the other hand, for the area with weak signal strength, the resolution of the ray is reduced by having a larger step size. Figure 1 demonstrates the process flow of the proposed adaptive 3D RT approach. The process flow is repeated until the whole area of interest is covered.

The complexity of adaptive 3D RT is low, as more rays are launched to an area with higher signal power. Because of the smaller ray launching step size at this region, better accuracy is obtained with higher numbers of received rays with high power level. Moreover, less computational time is needed as very few rays are launched to region with a high probability of weak signals. In the existing technique, rays are emitted to every direction, which contribute to a higher computational load to perform the intersection tests and other propagation calculations for all rays without any consideration to reduce the number of insignificant rays with a very low power level at the Rx or lost rays. However, the adaptive approach is much better in reducing the computational load due to its adaptive nature of ray launching based on strength of signal power. In the simulation, ray propagation mechanisms are combined which include transmission, diffractions and reflections before the ray hits the receptor [22]. The signal power strength, $E_{n}$ can be obtained by using (9).

$$
E_{n}=E_{i n}\left(Q_{n}\right)\left(\prod_{i=0}^{a_{n}} R_{i n} A_{r i n}\right)\left(\prod_{j=0}^{b_{n}} T_{j n} A_{t j n}\right)\left(\prod_{m=0}^{c_{n}} D_{m n} A_{d m n}\right) e^{-j k s_{n}}
$$

The terms $E_{i n}\left(Q_{n}\right), a_{n}, b_{n}, c_{n}, R_{i n}, T_{j n}$, and $D_{m n}$ are the initial power level, amount of reflections, amount of transmissions, amount of diffractions, reflection coefficient, transmission coefficient, and diffraction coefficient, respectively. The correlated spreading factors are denoted by $A_{\text {rin }}, A_{t j n}$, and $A_{d m n n} . S_{n}$ represents the traveled distance of a ray. Each Rx receives multiple rays due to direct path, diffraction, and reflection. At the receiver, total power level $E_{\text {total }}$ is the summation of power for each ray, which is given by (10).

$$
E_{\text {total }}=\sum_{n=0}^{M} E_{\text {in }}\left(Q_{n}\right)\left(\prod_{i=0}^{a_{n}} R_{\text {in }} A_{\text {rin }}\right)\left(\prod_{j=0}^{b_{n}} T_{j n} A_{t j n}\right)\left(\prod_{m=0}^{c_{n}} D_{m n} A_{d m n}\right) e^{-j k s_{n}}
$$

The total number of valid rays for each Rx is denoted by $M$. The transmitting signal power $P_{t}$, and antenna pattern, $Q_{n}$ is used to compute $E_{\text {in }}\left(Q_{n}\right)$ as given in (11).

$$
E_{\text {in }}\left(Q_{n}\right)=\frac{E_{0} \sqrt{G_{m}^{\prime}}}{p_{n}} \tilde{a}_{m}
$$


The expression $E_{0}=\sqrt{\frac{n_{0}}{4 \pi}} P_{t} G_{t}$ refers to the power level at reference distance of 1-meter from Tx. The term $n_{0}, G_{t}, G_{m}^{\prime}$, and $\tilde{a}_{m}$ refers to intrinsic impedance, gain of the antenna, antenna directivity, and polarization in the direction of $Q_{n}$, respectively. The $p_{n}$ is the distance between $Q_{n}$ and Tx. $V_{r n}$ is the voltage with respect to the Rx antenna and polarization which is derived using (12).

$$
V_{r n}=\sqrt{\frac{\lambda^{2} G_{r n} R_{0}}{4 \pi}}\left(\mathrm{E}_{n} \cdot \tilde{a}_{r m}\right) e^{j \phi}
$$

The term $\lambda, G_{r n}, R_{0}, \tilde{a}_{r m}$, and $e^{j \phi}$ are the wavelength, Rx gain, Rx impedance, Rx polarization, and fixedphase shift, respectively. The receiver power level is then computed using (13). The overall number of valid paths is represented by $M$.

$$
\begin{aligned}
& P_{r}=\frac{\left|\sum_{n=0}^{M} V_{m}\right|^{2}}{R_{0}}=\frac{\lambda^{2}}{4 \pi n_{0}}\left|\sum_{n=0}^{M}\left(E_{n} \cdot \tilde{a}_{r n}\right) \sqrt{G_{r n}}\right| \\
& =\frac{\lambda^{2}}{4 \pi n_{0}}\left|\sum_{n=0}^{M} \frac{E_{0} e^{-j k s_{n} \sqrt{G_{r n} G_{t n}^{\prime}}}}{P_{n}} \tilde{a}_{t n}\left(\prod_{i=0}^{a_{n}} R_{i n} A_{r i n}\right)\left(\prod_{j=0}^{b_{n}} T_{j n} A_{t j n}\right)\right|^{2} \\
& \left(\prod_{m=0}^{c_{n}} D_{m n} A_{d m n}\right) \tilde{a}_{r n}
\end{aligned}
$$

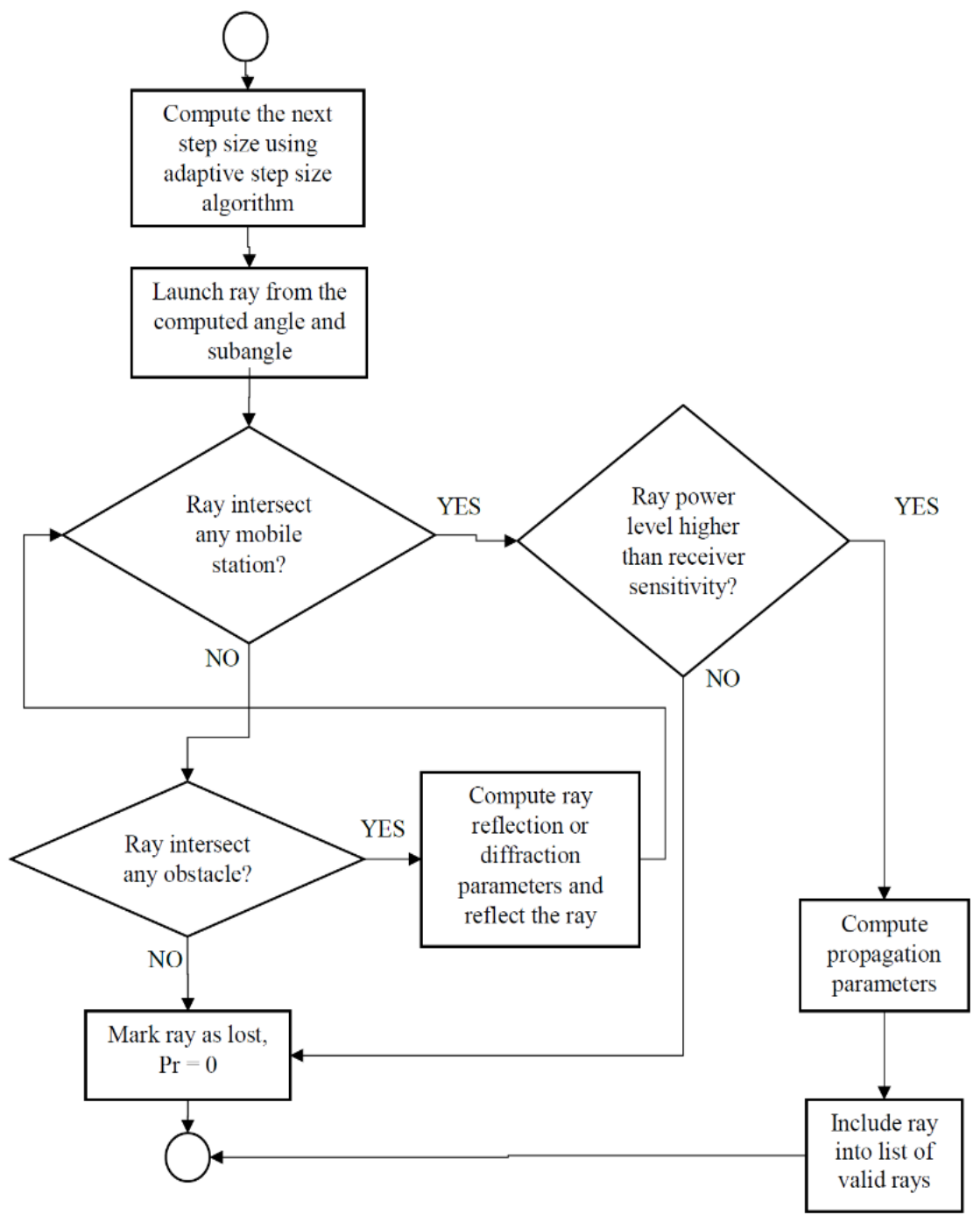

Figure 1. Process flow of the adaptive 3D RT method 


\section{VALIDATION OF RAY RACING RESULT}

In this research, the ray tracing simulation results were validated with the actual measurement data. The simulation layout was constructed according to the actual building where the measurement data were collected. The ground floor of the wireless communication center (WCC) building in Universiti Teknologi Malaysia (UTM) at Johor Bahru Campus in Malaysia was chosen as the venue for the measurement campaign to gather experimental data. The external walls of the building were made of concretes and bricks. The interior partitions for the main rooms were made using $5 \mathrm{~cm}$ gypsum board and thick glass. The doors and windows were made of wood, plastic, and transparent glass. The dimension of the floor was around $21 \mathrm{~m}$ by $30 \mathrm{~m}$.

Figure 2 shows the Tx and Rx setup for the measurement campaign. For Tx, an Agilent E4438C model signal generator was used together with the transmitting horn antenna with horizontal polarization and a beam width of 49 degrees. The height of the antenna is 1.5 meters and the gain is $14.5 \mathrm{~dB}$. For Rx, an Agilent E4440A model spectrum analyzer is used and connected with an omnidirectional antenna with the same height of 1.5 meter. The receiving antenna gain is $7 \mathrm{dBi}$. The Tx was fixed at one location in the tested area. For each measurement, a continuous wave $(\mathrm{CW})$ signal with frequency of $3.5 \mathrm{GHz}$ with power level of $10 \mathrm{dBm}$ was transmitted. The measurements of the received power at the Rx points were recorded by the spectrum analyzer starting from 1-meter distance between $\mathrm{Tx}$ and $\mathrm{Rx}$. The $\mathrm{Tx}$ and $\mathrm{Rx}$ antenna heights were maintained at 1.5 meters during the measurements, as an important factor in result accuracy [23].

For comparison purposes, the most used 3D conventional shooting and bouncing ray tracing (SBRT) technique and the proposed adaptive 3D RT method were selected in this simulation. The simulation results from both ray tracing techniques were analyzed and compared with actual measurement data. The layout for the RT simulations was constructed based on the actual WCC building layout using the in-house simulator. Figure 3 shows the visual representation of the simulation layout of WCC in 3D. The red dot is the Tx position and the blue dots are the tested Rx points.

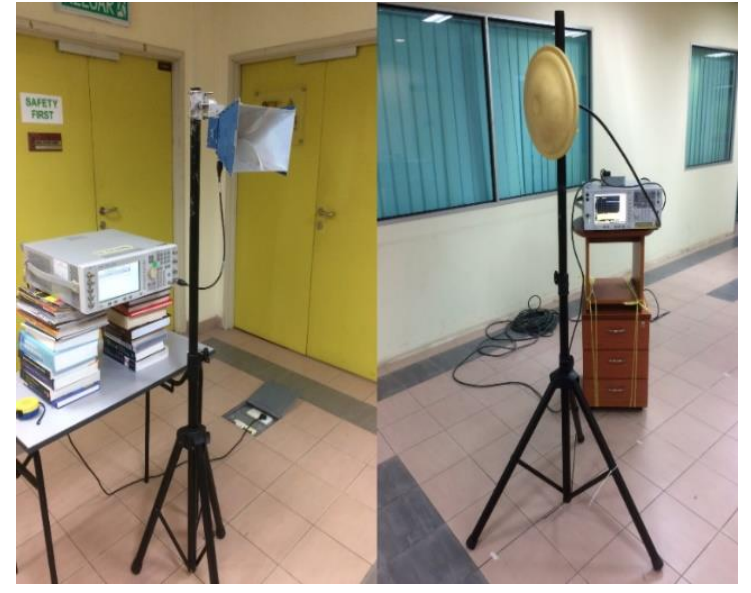

Figure 2. The Tx and Rx setup for measurement campaign

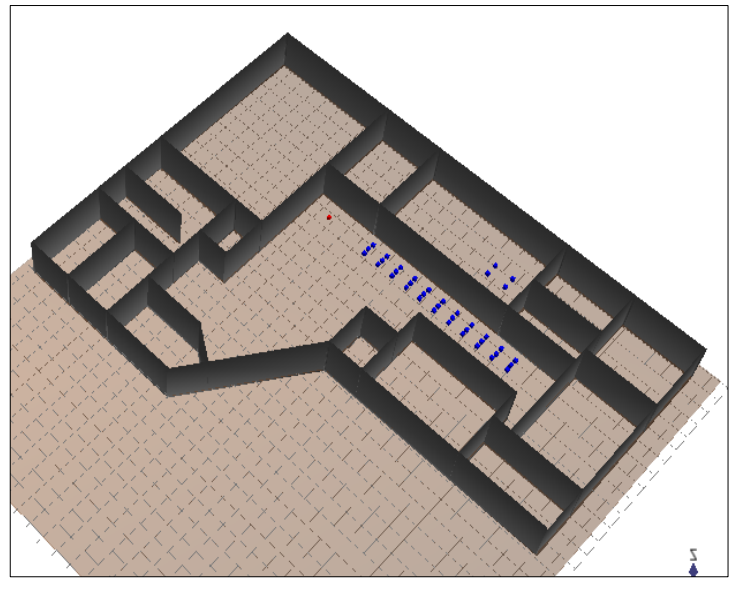

Figure 3. WCC layout in 3D for RT simulation

In the experiment, a sample of $20 \mathrm{Rx}$ points which includes the LoS and NLoS scenarios was tested. The power level and path loss were recorded for each point. For both RT techniques, the reports on the simulation results were generated in the simulator database which consists of information on the power level for each Rx point and the path loss.

Figure 4 shows the comparison graph of the power level at Rx points between simulation results and measurement data. The root mean square error (RMSE) was calculated using the standard formula to analyze the difference in power level between the measurement data and simulation results. The conventional shooting and bouncing ray tracing (SBRT) method showed a power level RMSE of 5.85, with respect to the experimental data. Among the 20 points, the receiver points $\mathrm{Rx} 4, \mathrm{Rx} 6, \mathrm{Rx} 7, \mathrm{Rx} 9$, and $\mathrm{Rx} 20$ expressed small differences in power level with the 3D SBRT approach. By using the adaptive 3D RT technique, as compared to measurement data, the power level RMSE was 2.55. For this method, most Rx points except Rx8 and Rx33 showed very small differences with the measurements. In any case, if the RMSE is small, the simulation result is very close to the practical implementation as shown in measurement data [24]. From the power level analysis, the proposed adaptive 3D RT technique showed a smaller RMSE value which is $3.3 \mathrm{~dB}$ lower than the existing 
SBRT method with respect to the measurements. It was proven that the proposed adaptive 3D RT technique had generated a result that is closer to the measurements as compared with the conventional SBRT approach.

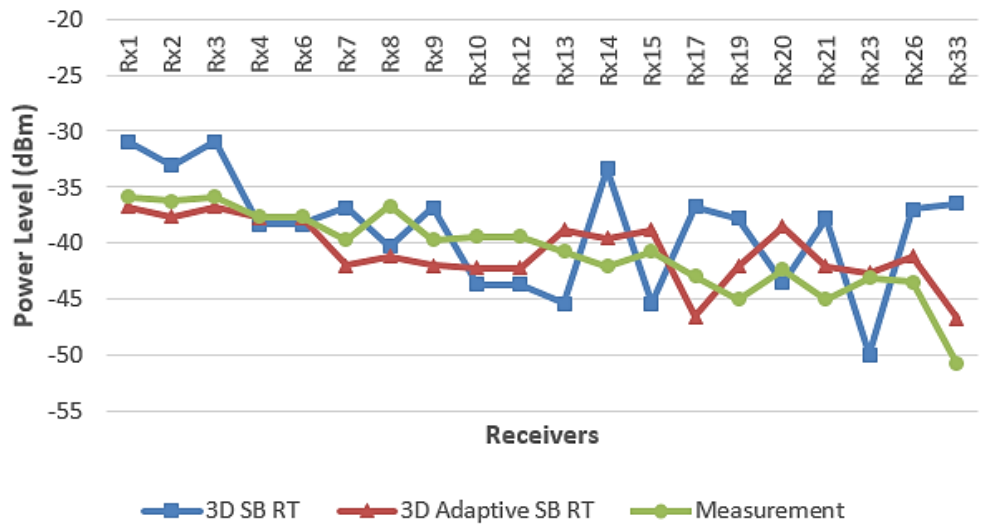

Figure 4. Power-level comparison graph between the measurements, 3D SBRT method, and proposed adaptive 3D RT method

Figure 5 shows the comparison graph of the number of rays received using the proposed adaptive 3D RT and the conventional 3D SBRT approaches. As the data on this parameter were not available from the measurement, the comparison was analyzed between the adaptive 3D RT method and the 3D SBRT data only. From Figure 5, the adaptive 3D RT method showed greater number of successful rays detected at the Rx points as compared with the conventional 3D SBRT technique. In RT approach, a higher level of accuracy for indoor propagation modeling can be achieved if a larger number of rays can be detected at the receivers. It is justified by the smaller value in power level RMSE for the adaptive RT method.

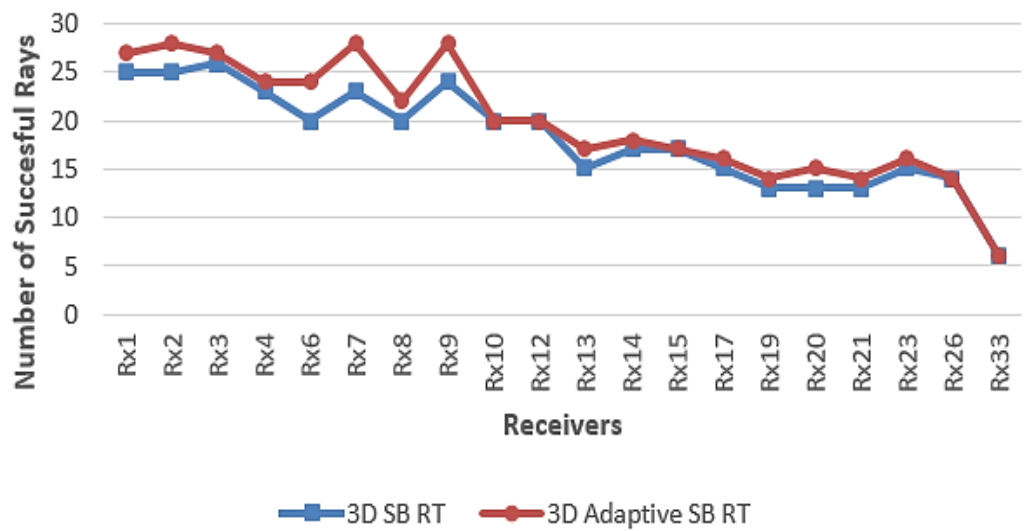

Figure 5. Number of rays received by the receiver: comparison between the conventional 3D SBRT and adaptive 3D RT methods

Figure 6 shows the comparison graph of simulation time for the conventional SBRT and adaptive 3D RT methods. In this case, both methods used the server with the same specification and configuration. From the findings, the conventional method required more computational time to perform the simulation compared to the adaptive 3D RT method. Since both methods were classified as site-specific propagation models, they can offer more precise radio signal predictions for a specific scenario than the stochastic or the empirical models. However, high computational time is identified as the main shortcoming for these 3D site-specific models [25]. In this finding, the adaptive 3D RT method saved about $44.4 \%$ of the time over the conventional method. It shows that the proposed adaptive 3D RT method is faster in generating the simulation results as compared with 3D SBRT approach, and thus mitigates the weakness of the site-specific models. 
In this study, we developed an enhanced ray launching algorithm with adaptive and flexible characteristics. By implementing an adaptive step size approach to launch more rays in the region with higher possibilities of strong signals as compared with other lesser probability region, it has greatly reduced the computational load and time which become the key contribution to this method. By analyzing the results, the proposed adaptive 3D RT technique showed a better improvement on simulation time, power level and coverage in modeling the radio wave propagation for the indoor scenario.

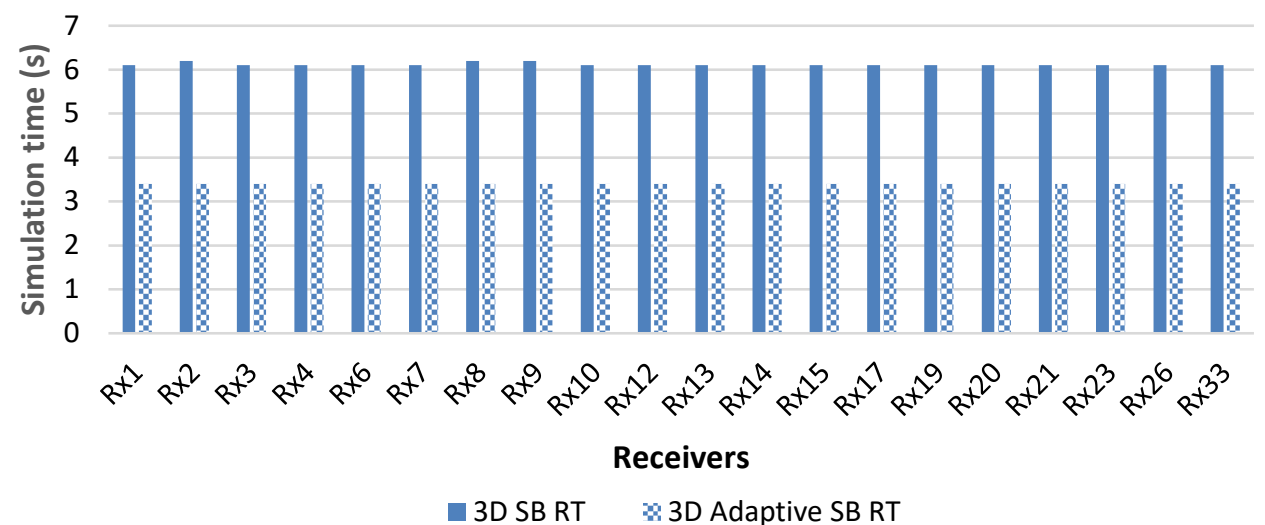

Figure 6. Comparison on simulation time between 3D SBRT and adaptive 3D RT approaches

\section{CONCLUSION}

In this paper, we performed an indoor radio propagation modeling at $3.5 \mathrm{GHz}$ by using the proposed adaptive 3D RT approach. This technique improves the ray launching algorithm by emitting more rays in an area with a high possibility of high power by adjusting the step size based on previously received power, which minimizes the computational complexity. The analysis on power level at multiple Rx points was compared and verified with measurement data. From the findings, the proposed adaptive 3D RT technique showed a smaller RMSE value than the existing 3D SBRT method with respect to the measurements. At the same time, the number of successful rays detected at the Rx points is higher by using the proposed method. In RT approach, a higher level of accuracy for indoor propagation modeling can be achieved if a larger number of rays can be detected at the receivers. This adaptive method also saved about $44.4 \%$ of the simulation time over the conventional method. It shows that the proposed adaptive 3D RT method is faster in generating the simulation results as compared to 3D SBRT approach, and thus mitigates the weakness of the site-specific models.

\section{ACKNOWLEDGEMENTS}

The authors thank Multimedia University and Wireless Communication Center-UTM for providing the financial and technical support for this research.

\section{REFERENCES}

[1] L. Azpilicueta, F. Falcone, and R. Janaswamy, "A hybrid ray launching-diffusion equation approach for propagation prediction in complex indoor environments," IEEE Antennas and Wireless Propagation Letters, vol. 16, pp. 214-217, 2017, doi: 10.1109/lawp.2016.2570126.

[2] F. Casino, L. Azpilicueta, P. Lopez-Iturri, E. Aguirre, F. Falcone, and A. Solanas, “Optimized wireless channel characterization in Large complex environments by hybrid ray launching-collaborative filtering approach," IEEE Antennas and Wireless Propagation Letters, vol. 16, pp. 780-783, 2017, doi: 10.1109/LAWP.2016.2604021.

[3] Y. Suzuki and M. Omiya, "Computer simulations for a site-specific modeling of indoor radio wave propagation," in 2016 IEEE Region 10 Conference (TENCON), Nov. 2016, pp. 123-126, doi: 10.1109/tencon.2016.7847972.

[4] F. Fuschini, E. M. Vitucci, M. Barbiroli, G. Falciasecca, and V. Degli-Esposti, "Ray tracing propagation modeling for future smallcell and indoor applications: A review of current techniques," Radio Science, vol. 50, no. 6, pp. 469-485, Jun. 2015, doi: 10.1002/2015RS005659.

[5] T. K. Geok et al., "A comprehensive review of efficient ray-tracing techniques for wireless communication," International Journal on Communications Antenna and Propagation (IRECAP), vol. 8, no. 2, Apr. 2018, Art. no. 123, doi: 10.15866/irecap.v8i2.13797.

[6] J. Medbo et al., "Radio propagation modeling for $5 \mathrm{G}$ mobile and wireless communications," IEEE Communications Magazine, vol. 54, no. 6, pp. 144-151, Jun. 2016, doi: 10.1109/mcom.2016.7498102.

[7] M. F. Iskander and Z. Yun, "Propagation prediction models for wireless communication systems," IEEE Transactions on 
Microwave Theory and Techniques, vol. 50, no. 3, pp. 662-673, Mar. 2002, doi: 10.1109/22.989951.

[8] S. Ju, Y. Xing, O. Kanhere, and T. S. Rappaport, "Millimeter wave and sub-terahertz spatial statistical channel model for an indoor office building," IEEE Journal on Selected Areas in Communications, vol. 39, no. 6, pp. 1561-1575, Jun. 2021, doi: 10.1109/jsac.2021.3071844.

[9] J. B. Andersen, T. S. Rappaport, and S. Yoshida, "Propagation measurements and models for wireless communications channels," IEEE Communications Magazine, vol. 33, no. 1, pp. 42-49, 1995, doi: 10.1109/35.339880.

[10] Z. Yun, Z. Zhang, and M. F. Iskander, "A ray-tracing method based on the triangular grid approach and application to propagation prediction in urban environments," IEEE Transactions on Antennas and Propagation, vol. 50, no. 5, pp. 750-758, May 2002, doi: 10.1109/tap.2002.1011243.

[11] F. Hossain et al., "A Smart 3D RT method: indoor radio wave propagation modelling at $28 \mathrm{GHz}$," Symmetry, vol. 11, no. 4, Apr. 2019, Art. no. 510, doi: 10.3390/sym11040510.

[12] M. S. Sarker, A. W. Reza, and K. Dimyati, "A novel ray-tracing technique for indoor radio signal prediction," Journal of Electromagnetic Waves and Applications, vol. 25, no. 8-9, pp. 1179-1190, Jan. 2011, doi: 10.1163/156939311795762222.

[13] F. Hossain et al., "An efficient 3-D ray tracing method: prediction of indoor radio propagation at $28 \mathrm{GHz}$ in $5 \mathrm{G}$ network," Electronics, vol. 8, no. 3, Mar. 2019, Art. no. 286, doi: 10.3390/electronics8030286.

[14] B. Bellekens, R. Penne, and M. Weyn, "Realistic indoor radio propagation for sub-GHz communication," Sensors, vol. 18, no. 6, Jun. 2018, Art. no. 1788 , doi: 10.3390/s18061788.

[15] A. I. Sulyman, A. T. Nassar, M. K. Samimi, G. R. Maccartney, T. S. Rappaport, and A. Alsanie, "Radio propagation path loss models for 5G cellular networks in the $28 \mathrm{GHZ}$ and $38 \mathrm{GHZ}$ millimeter-wave bands," \{IEEE\} Communications Magazine, vol. 52, no. 9, pp. 78-86, Sep. 2014, doi: 10.1109/mcom.2014.6894456.

[16] D. Shi, X. Tang, and C. Wang, "The acceleration of the shooting and bouncing ray tracing method on GPUs," in 2017 XXXIInd General Assembly and Scientific Symposium of the International Union of Radio Science (URSI GASS), Aug. 2017, pp. 1-3, doi: 10.23919/URSIGASS.2017.8105251.

[17] B. S. Lee, A. R. Nix, and J. P. McGeehan, "A spatio-temporal ray launching propagation model for UMTS pico and microcellular environments," in IEEE VTS 53rd Vehicular Technology Conference, Spring 2001. Proceedings (Cat. No.01CH37202), 2001, vol. 1, pp. 367-371, doi: 10.1109/VETECS.2001.944866.

[18] G. Durgin, N. Patwari, and T. S. Rappaport, "An advanced 3D ray launching method for wireless propagation prediction," in 1997 IEEE 47th Vehicular Technology Conference. Technology in Motion, vol. 2, pp. 785-789, doi: 10.1109/VETEC.1997.600436.

[19] Z. Yun and M. F. Iskander, "Ray tracing for radio propagation modeling: principles and applications," IEEE Access, vol. 3, pp. 1089-1100, 2015, doi: 10.1109/ACCESS.2015.2453991.

[20] D. M. Rose and T. Kurner, "An analytical 3D ray-launching method using arbitrary polygonal shapes for wireless propagation prediction," in 2014 IEEE 80th Vehicular Technology Conference (VTC2014-Fall), Sep. 2014, pp. 1-6, doi: 10.1109/VTCFall.2014.6965865.

[21] R. Luebbers, "Finite conductivity uniform GTD versus knife edge diffraction in prediction of propagation path loss," IEEE Transactions on Antennas and Propagation, vol. 32, no. 1, pp. 70-76, Jan. 1984, doi: 10.1109/TAP.1984.1143189.

[22] F. Hossain et al., "Indoor 3-D RT radio wave propagation prediction method: PL and RSSI Modeling validation by measurement at 4.5 GHz," Electronics, vol. 8, no. 7, Jul. 2019, Art. no. 750, doi: 10.3390/electronics8070750.

[23] Q. Hong et al., "The impact of antenna height on 3D channel: a ray launching based analysis," Electronics, vol. 7, no. 1, p. 2, Jan. 2018, doi: 10.3390/electronics7010002.

[24] F. Hossain, T. Geok, T. Rahman, M. Hindia, K. Dimyati, and A. Abdaziz, "Indoor millimeter-wave propagation prediction by measurement and ray tracing simulation at $38 \mathrm{GHz}$," Symmetry, vol. 10, no. 10, Oct. 2018, Art. no. 464, doi: 10.3390/sym10100464.

[25] H. Mi et al., "Implementation and evaluation of ray-tracing acceleration methods in wireless communication," in 2020 14th European Conference on Antennas and Propagation (EuCAP), Mar. 2020, pp. 1-5, doi: 10.23919/EuCAP48036.2020.9135370. 